\title{
The Exact Specification for Consumption of Blood- Pressure Regulating Drugs with a Numerical Model of Pulsatile Micropolar Fluid Flow in Elastic Vessel
}

\author{
[S. Maddah, H. Askarian, and S. Gholamrezaie]
}

\begin{abstract}
In the present paper, the problem of pulsatile micropolar blood flow through an elastic artery has been studied. An Arbitrary Lagrangian-Eulerian (ALE) formulation of the dominate equations have been produced to model the fully-coupled Fluid-Structure Interaction (FSI). Furthermore, those derived equations have also been numerically dealt with using finite difference scheme through exploiting a mesh generation technique which leads to a uniformly spaced grid in the computational plane. The effect of variations in cardiac output and elastic modulus of artery wall on blood pressure has been determined by utilizing blood-pressure regulating drugs like Atenolol. Also a numerical model has been prepared in order to precisely define and verify the effects of various dosage of a drug on blood flow in arteries without resorting to the inefficient prevalent experiments that could have many mistakes and costs. The numerical results have shown that the artery wall stiffness has been decreased about $29 \%$ by Atenolol dose between 0 and $0.02 \mathrm{mg} / \mathrm{kg}$ and have a blood pressure increment between 0.02 and $0.04 \mathrm{mg} / \mathrm{kg}$.
\end{abstract}

Keywords - Pulsatile blood flow, Atenolol, micropolar fluid, arbitrary Lagrangian-Eulerian, fluid structure interaction

\section{Introduction}

Nowadays Cardiovascular disorders have been recognized as one of the most prevalent fatality factors in all societies. The well-known risk factors causing such disorders are persistent hypertension (HTN). If the HTN problem is not treated thoroughly, it can shorten human life and this show the significance of our present studies. One of the wellknown drugs for HTN treatments is Atenolol, which was introduced in 1976 as a replacement for Propranolol. These drugs can have an impact on blood pressure by reducing heartbeat, cardiac output, and also changing the properties of artery walls.

Soroush Maddah, Assistant Professor in the Department of Mechanical Engineering,

Sari Branch, Islamic Azad University

Sari, Iran

Houra Askarian is Medical Doctor in the South Tehran Health Center, Tehran University of Medical Sciences,

Tehran, Iran

Sepehr Gholamrezaie is Bachelor Student of the Department of Mechanical Engineering,

Central Tehran Branch, Islamic Azad University,

Tehran, Iran
So far many experimental studies have been performed to identify the effects of increase in dosage of different drugs on heart rate and cardiac output. Some experiments were conducted on Atenolol in 1978 by Robinson and his collogues [1], on Propranolol in 1968 by Aström [2], Sowton and collogues [3] and finally on Prektolol in 1970 by Jewitt and collogues [4]. However in all those experiments, some sort of discontinuity and inaccuracy was noticed in the average changes of the obtained graphs in cardiac output, stroke volume, maximum $\mathrm{dP} / \mathrm{dt}$ of left ventricular and systemic vascular resistance against the above mentioned drags. Numerical modeling of blood flow in vessels can help to generate more accurate graphs and provide the average amount of hemodynamic quantities for all different doses of drugs.

The first person who figured out the motion equations for fully fluid filled elastic tube in 1914 might have been Witzing [5]. However the solution was limited to no viscous fluid flow. In 1957, Womersley obtained a harmonic solution for fluid and the wall in a flexible artery [6]. His work was the beginning of many further researches. He solved the linear Navier-Stokes equations for cylindrical elastic membrane with thin and isotropic wall analytically. In this model, the tube was filled with viscous fluid. After this study scientists have done many researches in order to pursue and develop Womersley theory. They exploited tubes with thick walls, materials with nonisotropic and viscoelastic features also by adding nonlinear terms to Navier-Stokes equations [7]. It is important to note that in classical fluid mechanics no description is considered for microstructure, but the particles of many fluids like animal blood and polymeric suspensions show microscopic effects like rotation. In 1999, Papautsky claimed that even for ordinary fluids like water in viscous liquids with small characteristic lengths, the microstructures play an important role [8]. Eringen propounded the micropolar fluid theory that accounts for internal structures of fluids $[9,10]$. He also showed that for the wide range of fluid flow, even for a fluid like water in micro-channels, the model of micropolar fluid flow is the best choice [11]. In this model, the microstructures of material have more maneuver room than classical flow. The microrotation vector has been be defined in such a way to allow the particles to rotate independently from the overall rotation and movement of the fluid. Extraction of dominating equations of flow with establishment of instant equilibrium in such element results in adding the angular momentum equations to the classical governing equations on fluid flow, i.e. linear momentum and 
Proc. of the Seventh International Conference On Advances in Mechanical, Aeronautical and Production Techniques - MAPT 2017 Copyright (C) Institute of Research Engineers and Doctors. All rights reserved.

ISBN: 978-1-63248-129-0 doi: 10.15224/ 978-1-63248-129-0-42

continuity equations.

Concerning the problem of blood flow in artery, the pulsatile blood flow can influence artery wall and cause its deformation. This factor can cause transformation of flow condition [12]. In scientific terminology this phenomenon is called "Fluid Structure Interaction (FSI)". For blood flow modeling in artery a two-way coupling method is required, because of the mutual effects between blood and artery wall the assumption of choosing small deformation has been proved according to a one-way coupling method which is incompatible with present problem.

Referring to numerical simulation of multidimensional problems, major distortions need to be overcome also an explicit illustration of Fluid-Structure Interaction should be presented. In order to create computer code generation to simulate such problems, the most important task is choosing an appropriate kinematical description for continuum. With this kind of choice, the relation of deformed continuum and meshes of computational zones is determined and the computer code is capable of verifying large distortions.

Often two classical descriptions of Lagrangian and Eulerian are used for the continuum mechanics [14] furthermore a description is developed for combining the advantages and minimizing the disadvantages of classical kinematical descriptions which is called the arbitrary Lagrangian-Eulerian (ALE) description. To figure out the present problem, the dominant equations on wall deformation need to be solved by Lagrangian formulation and the dominant equations on micropolar fluid flow need to be solved by ALE formulation.

The main topic in numerical solutions of partial differential equations is the grid generation. A wellconstructed grid can simplify the solution of a system of partial differential equations and remove the restrictions of finite-difference methods on solving the present problem. So a suitable mapping is employed to transform the physical plane into a computational domain with well-constructed grids.

Previous scientific studies on micropolar fluid were limited to the solution of pulsatile flow in rigid vessels [1517], but in the present paper, the ALE formulation for pulsatile micropolar fluid flow in elastic artery has been discretized to model the FSI problem for blood flow in artery. Also the numerical code of its transformed equations has been figured out.

\section{Blood Flow Model in ALE Formulation}

In the present study it is assumed that an incompressible micropolar fluid flow inside a cylindrical elastic tube has circular cross-section with radius of $R_{0}$ and length of $L$ (see Fig. 1). The coordinates of a material point in the cylindrical polar coordinates system are $(r, \theta, z)$ where the $\mathrm{z}$-axis is taken along the axis of the artery while $r, \theta$ are taken along the radial and the circumferential directions respectively and also the corresponding velocity and microrotation components are given by $(v, 0, u)$ and $(0, \omega, 0)$ respectively.

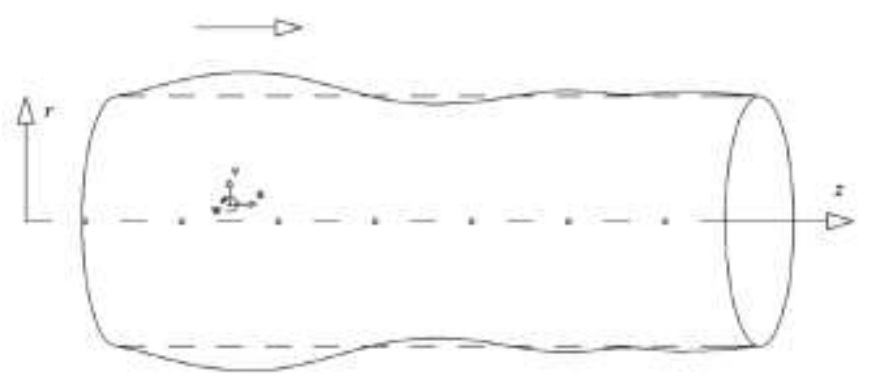

Fig. 1. The progressive pressure in micropolar fluid flow at a cylindrical elastic tube

The pulsatile inputs for a solution at the inlet flow rate of aortic artery have been taken from Kitney's measurements [18] and inlet velocity has been formulated with a sum of sinus functions as follows (also see Fig. 2):

$u=5.378 \times \sin \left\{0.08618\left(2 \pi f_{t} t+2.492\right)\right\}+2.645 \times \sin \left\{0.2367\left(2 \pi f_{t} t+5.042\right)\right\}+$ $0.4081 \times \sin \left\{0.5013\left(2 \pi f_{t} t+6.899\right)\right\}+0.01386 \times \sin \left\{1.714\left(2 \pi f_{t} t+1.062\right)\right\}+$ $0.0007281 \times \sin \left\{3.305\left(2 \pi f_{t} t+2.208\right)\right\}+0.001463 \times \sin \left\{2.839\left(2 \pi f_{t} t+2.088\right)\right\}-$ $0.0004833 \times \sin \left\{4.612\left(2 \pi f_{t} t+3.831\right)\right\}+0.0005284 \times \sin \left\{4.516\left(2 \pi f_{t} t+3.87\right)\right\}$

The governing equations with assumption of micropolar fluid flow without the body force and torque are given by [10]:

$$
\begin{aligned}
& \nabla \cdot \mathbf{v}=0 \\
& \rho \frac{\mathrm{D} \mathbf{v}}{\mathrm{D} t}=-\nabla p+(\mu+k) \nabla^{2} \mathbf{v}+k \nabla \times \mathbf{v}-k \nabla \times \boldsymbol{\omega} \\
& \rho J \frac{\mathrm{D} \boldsymbol{\omega}}{\mathrm{D} t}=\gamma \nabla^{2} \boldsymbol{\omega}+k \nabla \times \mathbf{v}-2 k \boldsymbol{\omega}
\end{aligned}
$$

Where $p$ is the hydrostatic pressure; $\mu$ and $k$ are the coefficients of shear and rotational viscosities respectively; $\gamma$ is the coupled viscosity, $\rho$ is the density of micropolar fluid and $J$ is the micro-inertia constant. To solve the problem with ALE formulation, the material velocity $\mathbf{V}$ should be replaced with the convective velocity $\boldsymbol{c}=\mathbf{v}-\hat{\mathbf{v}}$ in various terms of above equations. So:

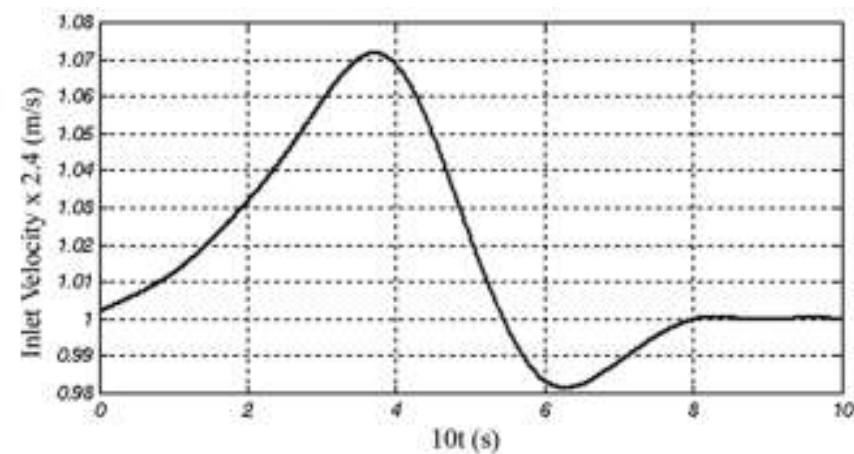

Fig. 2. Pulsatile time-averaged mean inlet velocity with a Doppler ultrasound (DUS) technique [18] 
Proc. of the Seventh International Conference On Advances in Mechanical, Aeronautical and Production Techniques - MAPT 2017 Copyright (C) Institute of Research Engineers and Doctors. All rights reserved.

ISBN: 978-1-63248-129-0 doi: 10.15224/ 978-1-63248-129-0-42

$\frac{\partial u}{\partial z}+\mathrm{St}\left(\frac{\partial v}{\partial r}+\frac{v}{r}\right)=0$

$\mathrm{St}\left[\frac{\partial u}{\partial t}+(v-\hat{v}) \frac{\partial u}{\partial r}\right]+(u-\hat{u}) \frac{\partial u}{\partial z}=-\frac{\partial p}{\partial z}+$

$\frac{1}{\operatorname{Re}}\left(\frac{\partial^{2} u}{\partial r^{2}}+\frac{1}{r} \frac{\partial u}{\partial r}+\frac{\partial^{2} u}{\partial z^{2}}\right)+\frac{R}{L_{C} \operatorname{Re}}\left(\frac{\partial \omega}{\partial r}+\frac{\omega}{r}\right)$

$\mathrm{St}\left[\frac{\partial v}{\partial t}+(v-\hat{v}) \frac{\partial v}{\partial r}\right]+(u-\hat{u}) \frac{\partial v}{\partial z}=-\frac{1}{\mathrm{St}} \frac{\partial p}{\partial r}+$

$\frac{1}{\operatorname{Re}}\left(\frac{\partial^{2} v}{\partial r^{2}}+\frac{1}{r} \frac{\partial v}{\partial r}+\frac{\partial^{2} v}{\partial z^{2}}-\frac{v}{r^{2}}\right)+\frac{1}{\operatorname{St} \operatorname{Re}} \frac{R}{L_{C}} \frac{\partial \omega}{\partial z}$

$J\left[\mathrm{St}\left[\frac{\partial \omega}{\partial t}+(v-\hat{v}) \frac{\partial \omega}{\partial r}\right]+(u-\hat{u}) \frac{\partial \omega}{\partial z}\right]=$

$\frac{m}{\operatorname{Re}}\left(\frac{\partial^{2} \omega}{\partial r^{2}}+\frac{1}{r} \frac{\partial \omega}{\partial r}+\frac{\partial^{2} \omega}{\partial z^{2}}-\frac{\omega}{r^{2}}\right)+$

$\frac{m^{2}}{\operatorname{Re}} \frac{R}{L_{C}}\left(\mathrm{St} \frac{\partial v}{\partial z}-\frac{\partial u}{\partial r}\right)-\frac{2 m}{\operatorname{Re}}\left(\frac{R}{L_{C}}\right)^{2} \omega$

Where $f$ is the pulsation frequency, $m$ is the Coupling Parameter[19] Re is the Reynolds number of micropolar fluid flow and St is the Strouhal number with following equations:

$m=\frac{k}{\mu+k} \quad, \quad \operatorname{Re}=\frac{\rho U R}{\mu+k} \quad, \quad \mathrm{St}=\frac{f R}{U}$

\section{Artery Model}

In this study it is assumed that the cylindrical wall of main arteries deforms linearly like homogeneous isentropic elastic shell with thickness $h$. If the radial displacements $\eta(z, t)$ for wall considered, the governing equation for wall deformation in Lagrangian coordinates results as following [20, 21]:

$f_{r}=\rho_{s} h \frac{\partial^{2} \eta}{\partial t^{2}}+\frac{h E}{1-v^{2}} \frac{\eta}{R^{2}}+p_{0}$

Here $f_{r}$ is the radial component of the contact force and $E, \rho_{s}$ and $v$ are the Young's modulus, shell density and Poisson ratio, respectively. The coupling between the fluid and the structure is obtained through the kinematic condition requiring continuity of the velocity evaluated at the deformed interface, so:

$$
u\left(R_{0}+\eta, z, t\right)=0 \quad, \quad v\left(R_{0}+\eta, z, t\right)=\frac{\partial \eta}{\partial t}
$$

And the dynamic condition requiring continuity of contact forces at the deformed interface:

$$
\left(\boldsymbol{\sigma}_{f} \cdot \boldsymbol{n}\right) \cdot \boldsymbol{e}_{r} \sqrt{\left(R_{0}+\eta\right)^{2}\left[1+\left(\frac{\partial \eta}{\partial z}\right)^{2}\right]}=f_{r}
$$

which $\sigma_{f}$ is the fluid stress tensor.

The equations (10) and (12) must be combined to use this model, and it results a law in any point of tube $p=p(R, x, t)$. This equation could be used for the pressure boundary condition.

\section{Numerical Method}

The following transformation can be used to transform a nonrectangular region in the physical plane into a rectangular region in the computational plane (also see Fig. 3):

$\xi=\frac{r}{R(z, t)} \quad, \quad z=z$

With these transformations, the governing equations will be produced. The projection method has been used for the satisfaction of continuity condition. This method results a Poisson equation that must be solved with other governing equations:

$$
\frac{1}{r} \frac{\partial p}{\partial r}+\frac{\partial^{2} p}{\partial r^{2}}+\frac{\partial^{2} p}{\partial z^{2}}=\delta\left[\frac{\partial \tilde{u}}{\partial z}+\mathrm{St}\left(\frac{\partial \tilde{v}}{\partial r}+\frac{\tilde{v}}{r}\right)\right]
$$

where $\delta=\frac{R}{L}=\frac{R}{U \Delta t}$ is the displacement parameter.

The equation (14) uses the pressure boundary conditions on wall that results pressure field in computational domain. The governing equations on fluid flow (6-8) and the continuity equation (16) shall be transformed to computational domain with regular meshes to reach the reasonable numerical solution. It is noticeable that this method must be done for all of the other boundary conditions. Then the finite difference method has been used for discretizing equations.

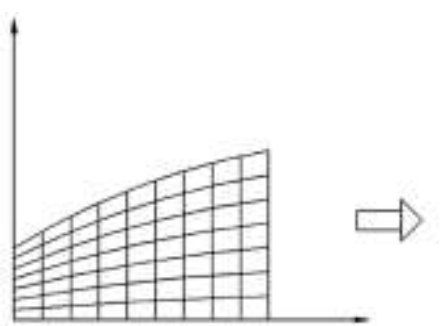

a

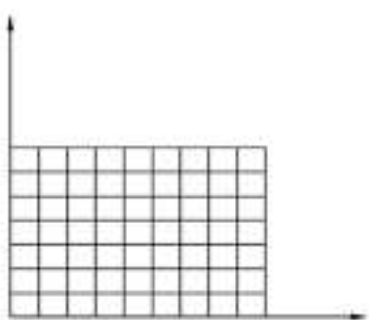

$\mathrm{b}$
Fig. 3. Description of Physical plane a) Physical plane; b) computational plane 
Proc. of the Seventh International Conference On Advances in Mechanical, Aeronautical and Production Techniques - MAPT 2017 Copyright (C) Institute of Research Engineers and Doctors. All rights reserved.

ISBN: 978-1-63248-129-0 doi: 10.15224/ 978-1-63248-129-0-42

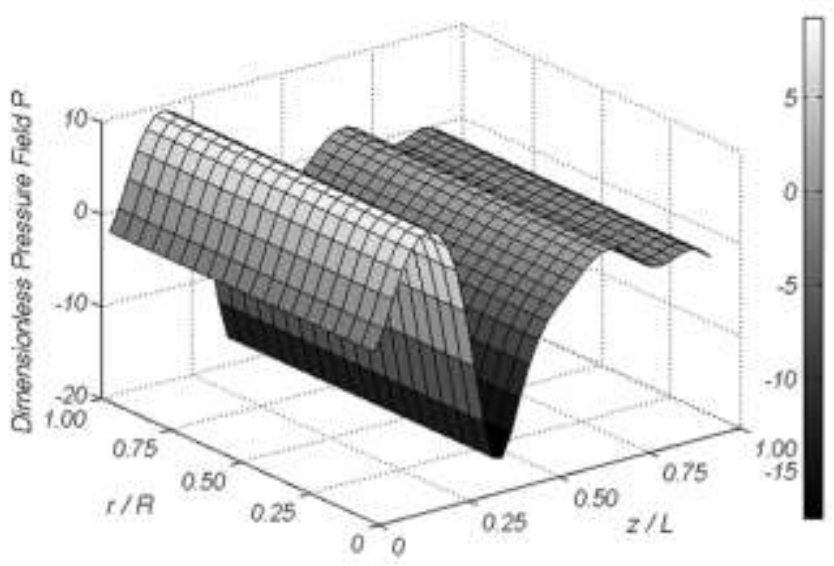

Fig. 4. Pressure field at a specified time

\section{v. Results and Discussion}

The mentioned code has been solved and the results for pressure field have been shown in Fig. 4. For modeling and defining the pressure variations with changing of Atenolol dosage, the results are received from numerical solution and calibrate with existing results for heartbeat, wall resistance and mean Aortic pressure variations with three different dosages of Atenolol consumption in Robinson et al. work [1] .From this study a graph provided to determine the exact dosage of Atenolol.

In Table I, Robinson et al. have presented the results of their studies and measurements. The aortic pressure was measured with a fluid filled manometer and cardiac outputs were determined by the Indocyanine Green Dye Dilution method with a Gilford densitometer [1]. These results have been used in numerical code and the module of elasticity changed to gain the above pressures in any case. Table II shows magnitude of module of elasticity for different dosage of Atenolol.

TABLE I. The Hemodynamic EFFects Of GRAdEd Doses OF ATENOLOL [1]

\begin{tabular}{ccccc}
\hline \hline $\begin{array}{c}\text { Dose of } \\
\text { Atenolol } \\
(\mathrm{mg} / \mathrm{kg})\end{array}$ & Control & 0.03 & 0.06 & 0.12 \\
\hline $\begin{array}{c}\text { Heart rate } \\
\text { (beats/min) }\end{array}$ & $69 \pm 2$ & $62 \pm 2$ & $61 \pm 2$ & $60 \pm 3$ \\
$\begin{array}{c}\text { Cardiac } \\
\text { output }(1 / \mathrm{min})\end{array}$ & $4 \pm 0.3$ & $3.4 \pm 0.2$ & $3.1 \pm 0.2$ & $2.9 \pm 0.3$ \\
$\begin{array}{c}\text { Aortic mean } \\
\text { pressure } \\
(\mathrm{mmHg})\end{array}$ & $89 \pm 6$ & $91 \pm 6$ & $89 \pm 4$ & $85 \pm 4$ \\
\hline \hline
\end{tabular}

TABLE II. The Results OF NumericAl Code For Module OF ELASTICITY

\begin{tabular}{ccccc}
\hline \hline $\begin{array}{c}\text { Dose of Atenolol } \\
(\mathrm{mg} / \mathrm{kg})\end{array}$ & Control & 0.03 & 0.06 & 0.12 \\
\hline $\begin{array}{c}\text { Module of } \\
\text { Elasticity (M pa) }\end{array}$ & 0.401 & 0.282 & 0.276 & 0.275 \\
\hline \hline
\end{tabular}

The accompaniment of flow rate and wall properties variations with dosage of Atenolol doesn't give a linear variation pattern of pressure in different dosage of drug. So the existing experimental results couldn't be applied for exact prescription, because they are discontinuous for some dosage of consumption but the comparison of the results in Table II with module of elasticity of blood artery [22] shows that the dose-response curves are nonlinear. However the dosage of Atenolol effects on magnitude of cardiac output, blood flow rate of mean Aortic pressure and also module of elasticity. The dose-pressure and dose-cardiac output curves can be measured experimentally that exist in many researches. We used the results of measurements for dosepressure in consumption of Atenolol [23] and the results of measurements for dose-cardiac output from Svendsen et al. paper [24] (Table III) in our numerical code and produced a continuous dose-elasticity module curve for consumption of Atenolol (Fig. 5).

TABLE III. CARDIAC INDEX BEFORE AND AFTER INTRAVENOUS ADMINISTRATION OF INCREASING DOSES OF ATENOLOL (X \pm SEM) [24]

\begin{tabular}{|c|c|c|c|c|c|c|}
\hline $\begin{array}{c}\text { Doses of Atenolol } \\
\text { (mg/kg body weight) }\end{array}$ & 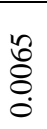 & 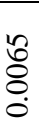 & $\stackrel{1}{0}$ & 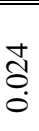 & $\begin{array}{l}\infty \\
\stackrel{0}{0} \\
0\end{array}$ & ஓ̆ \\
\hline $\begin{array}{c}\text { Cardiac Output } \\
\text { Control: } 2.87 \pm 0.26 \\
\quad\left(1 / \mathrm{min} / \mathrm{m}^{2}\right)\end{array}$ & $\begin{array}{l}\text { ป̂ } \\
0 \\
+1 \\
\text { J } \\
\text { ¿ }\end{array}$ & $\begin{array}{l}\text { ํ. } \\
0 \\
+1 \\
\circ \\
\curvearrowleft \\
\text { ণ }\end{array}$ & 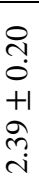 & 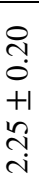 & $\begin{array}{l}\bar{y} \\
0 \\
+1 \\
\tilde{D} \\
\text { तi }\end{array}$ & $\begin{array}{l}\vec{N} \\
0 \\
+1 \\
0 \\
\text { ㅁ }\end{array}$ \\
\hline
\end{tabular}

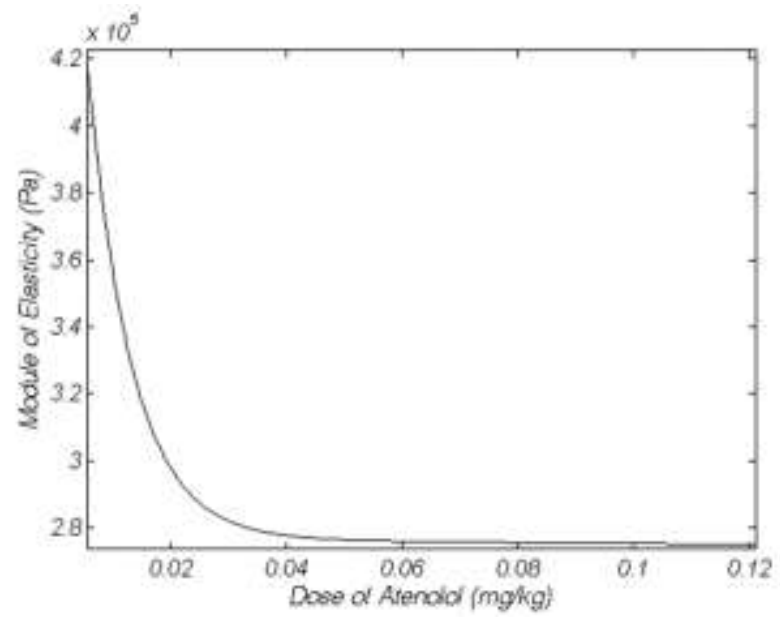

Fig. 5. Results of numerical code for module of elasticity in different dose of Atenolol

The derivation of curves shows that how the properties of artery wall and the outlet flow rate vary with using of different dosage of Atenolol drug. Also the following dosepressure diagram from numerical code can help physicians to prescript precise dosage of Atenolol. 


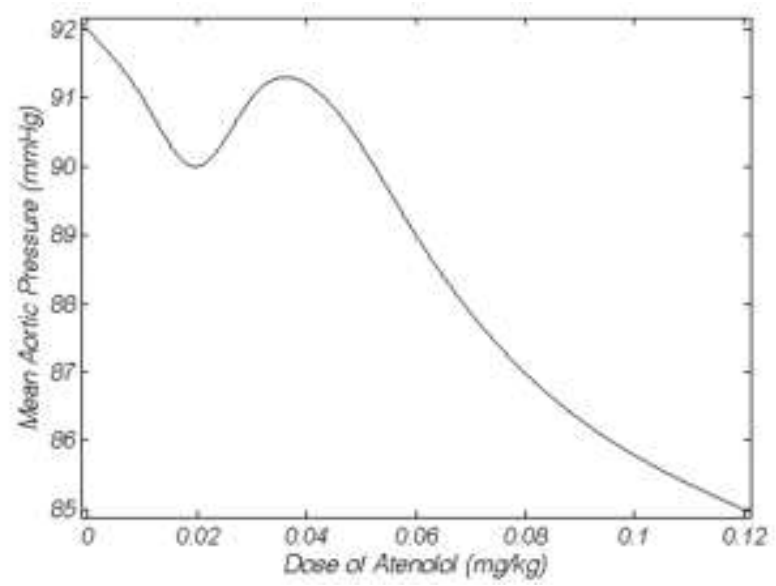

Fig. 6. Dose-pressure results of Atenolol consumption from numerical code

This diagram (Fig. 6) illustrates that the double dosage selection can decrease the blood pressure until more than two times and also can increase the pressure. By comparing dose-pressure diagram with dose-elasticity module diagram it is concluded that between 0 to $0.02 \mathrm{mg} / \mathrm{kg}$ Atenolol dose, the pressure magnitude decreased about $2 \%$, while the elasticity module declines about $29 \%$. But the decrement of elasticity module in the range of 0.02 to 0.12 is about $7 \%$. At first this order results that the pressure magnitude almost be invariant or even it has a low growth between 0.02 and 0.04 . Also, it shows a $7 \%$ decrement for 0.04 to 0.12 Atenolol doses that is accompanied with more reduction heart rate and cardiac output.

\section{Conclusions}

HTN is one of the most important diseases in the world and has many side effects on different organs like kidney, brain, etc. When a non-emergent patient with long-term signs of HTN refers to physician, the doctor checks blood pressure (Bp) in two visits if the measured Bps be more than $140 / 90 \mathrm{mmHg}$, diagnosis is HTN. Atenolol is one of the most widely used drugs and the first-line treatments for HTN. The initial dose of Atenolol is one tablet a day. If after two weeks patient's Bp doesn't come to normal, the dosage could increase to two tablets a day. But it doesn't mean that if the dosage increased from one to two tablets, it has the satisfactory result on the patient. It may cause bradycardia (slow heart rate) and hypotension with shock. So, dosage regulating of Atenolol or other anti-hypertensive drugs are very important, thus, it is very useful to find a suitable method to show relation between dosage of drugs like Atenolol and their effects on pressure. The numerical code for blood flow and the graphs that have been produced in this paper are very valuable for pharmacy. Because of a new drug production for blood pressure regulating needs numerous experiments for observation of its effects on hemodynamic quantities in arteries that has many mistakes and costs.

\section{References}

[1] C. Robinson, G. Jackson, C. Fisk and D. Jewitt, "Haemodynamic effects of Atenolol in patients with coronary artery disease," British Heart Journal, vol. 40, pp. 22-28, 1978.
[2] H. Aström, "Haemodynamic effects of beta-adrenergic blockade," British Heart Journal, vol. 30, pp. 44-49, 1968.

[3] E. Sowton, R. Balcon, D. Cross and H. Frick, "Haemodynamic effects of ICI 50172 in patients with ischaemic heart disease," British Medical Journal, vol. 1, pp. 215-216, 1968.

[4] D. E. Jewitt, P. A. Burgess and J. P. Shillingford, "The circulatory effects of practolol (ICI 50172) in patients with acute myocardial infarction," Cardiovascular Research, vol. 4, pp. 188-193, 1970.

[5] K. Witzing, "Uber erzwungene wellenbewgungen zaher inkompressibilen flüssigkeiten in elastischen rohren," Inaugural Dissertation, University of Bern, 1914.

[6] J. R. Womersley, "An elastic tube theory of pulse transmission and oscillatory flow in mammalian arteries," Wright Air Development Centre, Technical Report, (614-56), 1957.

[7] R. H. Cox, "Comparison of linearized wave propagation models for arterial blood flow analysis," ASME Trans., vol. 2, pp. 1-11, 1969.

[8] I. Papautsky, J. Brazzle, T. Ameel and A. B. Frazier, "Laminar fluid behaviour in microchannels using micropolar fluid behavior," Sens. and Act., vol. 73, pp. 101-108, 1999.

[9] A. C. Eringen, "Simple Microfluids," Int. J. Eng. Sci., vol. 2, pp. 205217, 1964.

[10] A. C. Eringen, "Theory of Micropolar Fluids," J. Math. Mech., vol. 16, pp. 1-18, 1966.

[11] C. K. Kang and A. C. Eringen, "The effect of microstructure on the rheological properties of blood," Bulletin of Mathematical Biology, vol. 38, pp. 135-159, 1976.

[12] G. Papadakis, "A novel pressure-velocity formulation and solution method for fluid-structure interaction problems," Journal of Computational Physics, vol. 227, pp. 3383-3404, 2008.

[13] K. Hassani, M. Navidbakhsh and M. Rostami, "Simulation of the cardiovascular system using equivalent electronic system," Biomed. Pap. Med. Fac. Univ. Palacky. Olomouc. Czech Repub., vol. 150, no. 1, pp. 105-112, 2006.

[14] L. W. Malvern, "Introduction to the Mechanics of a Continuous Medium," Prentice-Hall, Englewood Cliffs, 1969.

[15] A. Moosaie and GH. Atefi, "A Cosserat Continuum Mechanical Approach to Steady Flow of Blood through Artery," Journal of Dispersion Science and Technology, vol. 28, pp. 765-768, 2007.

[16] A. Moosaie and GH. Atefi, "Analysis of concentrated suspension flow by utilizing a Cosserat-type continuum theory," Journal of Dispersion Science and Technology, vol. 28, pp. 901-906, 2007.

[17] S, Khani and GH. Atefi, "A Semi-Analytical Semi-Experimental Solution for Flow Simulations in Cosserat Continuum Mechanical Approach to Pulsatile Blood Flow in a Tapered Femoral Artery of a Dog," Journal of Dispersion Science and Technology, vol. 31, pp. 1489-1494, 2010

[18] R. Kitney and D. Giddens, "Analysis of blood velocity waveforms by phase shift averaging and autoregressive spectral estimation," Journal of Biomechanical Engineering, vol. 105, pp. 398-401, 1983.

[19] S. C. Cowin, "Polar fluids," Phys Fluids, vol. 11, no. 9, pp. 19191927, 1968

[20] P. G. Ciarlet and V. Lods, "Asymptotic analysis of linearly elastic shells: Justification of membrane shell equations," Arch Rational Mech Anal., vol. 136, no. 2, pp. 119-161, 1996.

[21] P. G. Ciarlet, "Mathematical Elasticity Vol. III: Theory of Shells," Amsterdam: Stud Math Appl 29, North-Holland, 2000.

[22] T. J. Pedly, "The Fluid Mechanics of Large Blood Vessels," Cambridge: Cambridge University Press, 1980.

[23] A. J. Marshall, D. W. Barritt, S. Heaton and J. D. Harry, "Dose response for blood pressure and degree of cardiac B-blockade with Atenolol," Postgraduate Medical Journal, vol. 55, pp. 537-540, 1979.

[24] T. L. Svendsen, O. J. Hartling, J. Trap-Jensen, A. McNair and J. Bliddal, "Adrenergic beta receptor blockade: Hemodynamic importance of intrinsic sympathomimetic activity at rest," Clin. Pharmacol. Ther., vol. 29, no. 6, pp. 711-718, 1981.

About Corresponding Author:

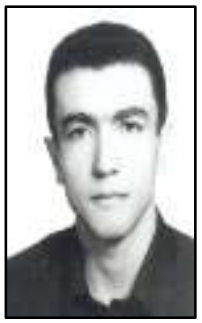

Soroush Maddah is Assistant Professor and Head of Mechanical Engineering Department, Sari Branch, Islamic Azad University, Sari, Iran

Phone: +98-9111558085;

Fax: +98-2188533147; 